\title{
Flecainide Enhances Endocardial Unipolar Voltage Abnormalities in Brugada Syndrome
}

\author{
Dimitrios Asvestas, Antigoni Sakellaropoulou, Panagiotis Mililis, Michael Efremidis, Antonios Sideris and \\ Konstantinos P Letsas
}

Second Department of Cardiology, Laboratory of Cardiac Electrophysiology, "Evangelismos" General Hospital of Athens, Athens, Greece

\begin{abstract}
A 40-year-old male patient with Brugada syndrome underwent high-density endocardial voltage mapping (>1000 points) of the right ventricular outflow tract (RVOT) using a three-dimensional mapping system. Electroanatomical maps were performed before and after flecainide infusion $(70 \mathrm{mg}$ ), setting the reference value for normal unipolar electrograms at $>4 \mathrm{mV}$. A broader area of abnormal unipolar signals was developed at the free wall of the RVOT after flecainide infusion. Endocardial unipolar mapping after sodium channel challenge may accurately identify the epicardial arrhythmogenic substrate and may have potential clinical implications in endocardial ablation strategies.
\end{abstract}

\section{Keywords}

Brugada syndrome, electroanatomical mapping, voltage mapping, ventricular fibrillation

Disclosure: Dimitrios Asvestas, Antigoni Sakellaropoulou, Panagiotis Mililis, Michael Efremidis, Antonios Sideris and Konstantinos $\mathrm{P}$ Letsas have nothing to declare in relation to this article.

Review Process: Double-blind peer review.

Authorship: All named authors meet the criteria of the International Committee of Medical Journal Editors for authorship for this manuscript, take responsibility for the integrity of the work as a whole and have given final approval for the version to be published.

open Access Statement: This article is published under the Creative Commons Attribution Noncommercial License, which permits any non-commercial use, distribution, adaptation and reproduction provided the original author(s) and source are given appropriate credit. (C) The Authors 2018.

Compliance with Ethics: All procedures were followed in accordance with the responsible committee on human experimentation and with the Helsinki Declaration of

1975 and subsequent revisions. Informed consent was received from the patient involved in this case study.

Received: 18 May 2018

Accepted: 24 July 2018

Citation: European Journal of Arrhythmia \& Electrophysiology, 2018;4(2):75-6

Corresponding Author: Dimitrios Asvestas, Second Department of Cardiology, Laboratory of Cardiac Electrophysiology, Evangelismos Genera Hospital of Athens, 10676, Athens, Greece E: dimasvestas@gmail.com

Support: No funding was received in the publication of this article.
Brugada syndrome (BrS) is a genetic arrhythmia syndrome with increased risk of sudden cardiac death. Unmasking a type I BrS electrocardiogram (ECG) pattern after administration of sodium channel blockers may be associated with an increase in epicardial substrate abnormalities. We hypothesised that high-density endocardial unipolar voltage mapping of the right ventricular outflow tract (RVOT), before and after flecainide infusion in a patient with BrS, may detect enhancement of the electroanatomical abnormalities possibly related to the modification of the abnormal epicardial tissue.

A 40-year-old male asymptomatic patient with Brs was referred to our centre and underwent electrophysiological study for risk stratification via programmed ventricular stimulation. Initially, he underwent high-density endocardial voltage mapping of the RVOT during sinus rhythm. Cardiac-magnetic resonance imaging ruled out structural heart disease. More than 1,000 points were sampled throughout the RVOT using a multi-electrode mapping catheter. The reference value for normal unipolar electrograms was set at $>4 \mathrm{mV}$ and electroanatomical maps were performed before and after flecainide infusion (70 mg). As shown in Figure 1, the patient displayed a small area of unipolar voltage abnormalities beneath the pulmonary valve (Figure 1A). After administration of flecainide, a Brugada ECG pattern with typical coved ST elevation $>2 \mathrm{~mm}$ in right precordial leads is seen (Figure 2), and a broader area of abnormal unipolar signals was developed at the free wall of the RVOT (Figure 1B). Programmed ventricular stimulation was performed from the right ventricle, inducing ventricular fibrillation (VF) (Figure 3). Endocardial unipolar mapping after sodium channel challenge may accurately identify the epicardial arrhythmogenic substrate and may have potential clinical implications in endocardial ablation strategies.

The endocardial unipolar voltage abnormalities possibly reflect the epicardial bipolar voltage abnormalities that have been detected at the RVOT of patients with BrS. ${ }^{12}$ Venlet et al. have shown that the optimal endocardial unipolar voltage cut-off for the identification of epicardial right ventricular scar was $3.9 \mathrm{mV}$ in patients who underwent endocardial and epicardial right ventricular voltage mapping. ${ }^{3}$ Brugada et al. elegantly demonstrated a significant increase in low bipolar voltage epicardial areas in RVOT after flecainide infusion. ${ }^{2}$ Flecainide facilitates the identification of the extension and distribution of arrhythmia substrate during epicardial mapping. ${ }^{2}$ Epicardial substrate elimination was associated with normalisation of BrS ECG pattern and the absence of ventricular tachycardia/VF inducibility. In addition, an endocardial ablation approach has been shown to normalise the ECG and to supress VF storms. ${ }^{4}$ Endocardial unipolar mapping after sodium channel challenge may accurately identify the epicardial arrhythmogenic substrate and may have potential clinical implications in endocardial ablation strategies. $\square$ 
Figure 1: Electroanatomical mapping before and after flecainide infusion

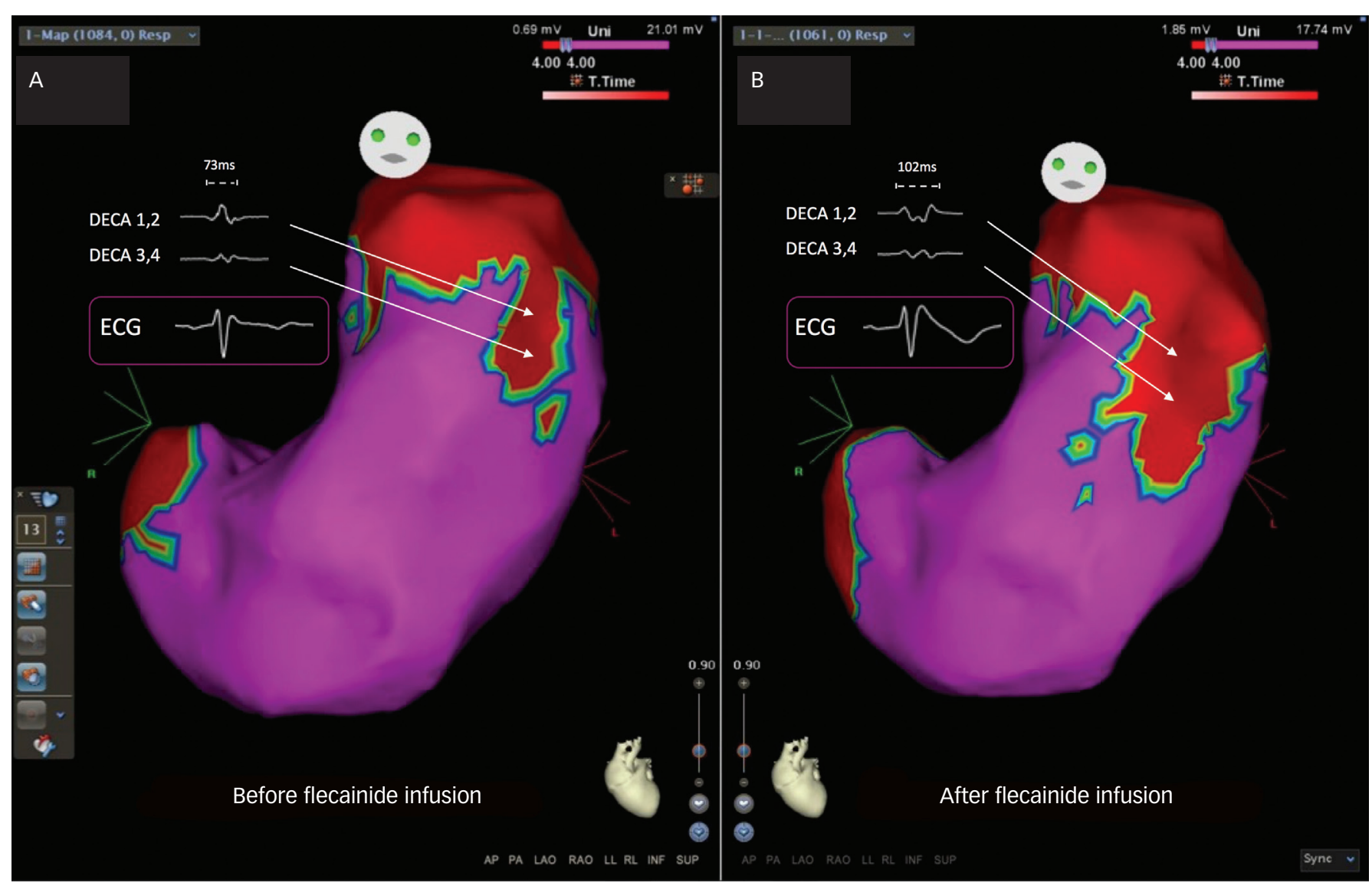

A broader area of abnormal unipolar electrograms was developed at the free wall of the RVOT after $70 \mathrm{mg}$ of flecainide infusion (A). Similarly, the multipolar catheter DecaNav ${ }^{*}$ (Biosense-Webster Inc., Irvine, CA, US) recorded abnormal multicomponent bipolar signals after drug challenge (B). The ECG (lead $V_{2}$ positioned at the third ICS) revealed a coved type ST segment elevation. DECA = DecaNAV; ECG = electrocardiogram; RVOT = right ventricular outflow tract.

Figure 2: Electrocardiographic changes after flecainide infusion

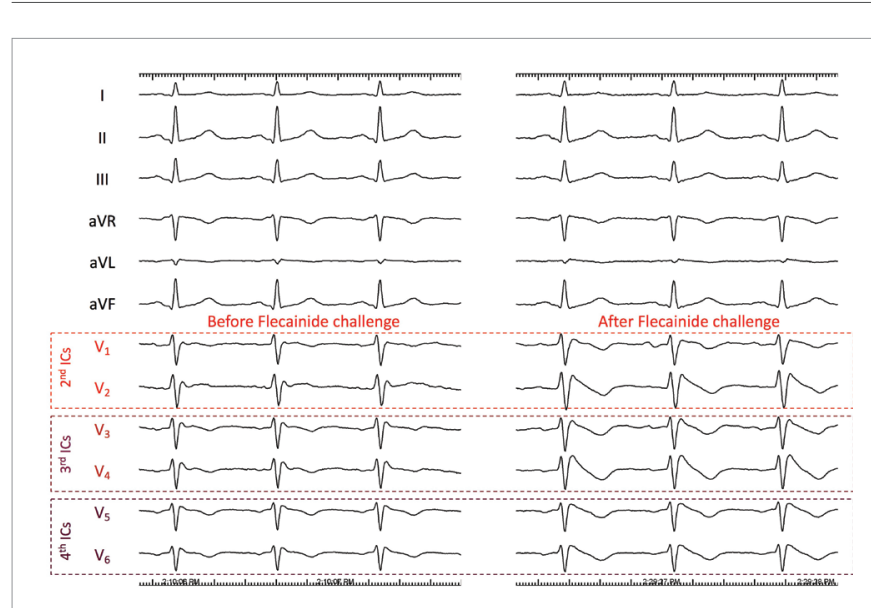

Precordial leads were positioned at the second $\left(V_{1}-V_{2}\right)$, third $\left(V_{3}-V_{4}\right)$ and fourth $\left(V_{5}-V_{8}\right)$ ICS. A coved ST-T segment elevation $>2 \mathrm{~mm}$ was unmasked after $70 \mathrm{mg}$ of flecainide infusion. ICS = intercostal space.
Figure 3: Induction of ventricular fibrillation during programmed stimulation
Programmed ventricular stimulation was performed from the right ventricle, inducing ventricular fibrillation at drive train with a $500 \mathrm{~ms}$ cycle length and two extra stimuli (500-230-250 ms).

Letsas KP, Efremidis M, Vlachos K, et al. Right ventricular outflow tract high-density endocardial unipolar voltage mapping in patients with Brugada syndrome: evidence for electroanatomical abnormalities. Europace. 2018;20:557-63.

Brugada J, Pappone C, Berruezo A et al. Brugada syndrome phenotype elimination by epicardial substrate ablation. Circ Arrhythm Electrophysiol. 2015:8:1373-81.

3. Venlet J, Piers SRD, Kapel GFL, et al. Unipolar endocardial voltage mapping in the right ventricle: Optimal cutoff values correcting for computed tomography-derived epicardial fat thickness and their clinical value for substrate delineation. Circ Arrhythm Electrophysiol. 2017;10:1-8.

4. Hayashi T, Nitta J, Goya M, et al. Endocardial only catheter ablation with substantial improvement in ventricular fibrillation recurrences in a patient with Brugada syndrome. HeartRhythm Case Rep. 2016;2:428-31. 